MODELING, IDENTIFICATION AND CONTROL, 2003, VOL. 24, NO. 2, 71-85

doi:10.4173/mic.2003.21

\title{
Analysis of Vortex Induced Vibrations of Marine Risers
}

\author{
KARL E. KAASEN* and HALVOR LIE* \\ Keywords: Vortex Induced Vibration, Marine Riser, Modal Analysis, Least \\ Squares Estimation, Fast Fourier Transformation
}

\begin{abstract}
Vortex induced vibrations (VIV) can be a severe problem to marine risers with regard to fatigue damage and drag loading. In order to design marine risers, therefore, it is necessary to have good theoretical and numerical models for prediction of VIV. Full-scale data are needed for verification of the models. To this end, three drilling risers were instrumented with accelerometers and rotationrate devices for measurement of VIV. Also, sea current was measured at number of depths for reference. A large quantity of data was collected during the time the instrument system was in operation. In order to choose data records for further investigation and comparison with theoretical models the raw data had to be conditioned and converted to a suitable form.

The paper describes how the riser lateral displacements were derived from the measurements. A major task has been to rid the acceleration measurements of the influence of gravity due to the riser's rotations out of the vertical and include the measurements of angular motion in a consistent way. This has been done using modal decomposition and a least-squares method combined with frequencydomain calculation to estimate the modal weights. MATLAB was used for the calculations and the presentation of results. An example of results is given.
\end{abstract}

\section{Introduction}

Slender marine structures such as marine cables, pipelines and risers may be set in oscillation by a constant current. The excitation is caused by vortices that are shed alternatingly from either side of the structure, hence the name vortex induced vibration (VIV). For marine structures VIV is important with respect to fatigue damage. Also VIV may cause an increase in the current drag on the riser. For design of marine cables and risers it is therefore important to have models that can predict VIV with realism.

The phenomenon of VIV is described extensively in the literature, see Blevins (1990) and Vandiver (1993). For a fixed, rigid circular cylinder in a uniform flow directed normally to its axes the vortex shedding frequency is

$$
f_{s}=S t \frac{U}{D}
$$

where $U$ is the free stream speed and $D$ is the cylinder diameter. The Strouhal number, $S t$, is a function of the Reynolds number (Sheppard and Omar, 1992). In the subcritical range $\left(300<R e<1.5 \times 10^{5}\right) S t$ varies little and has a value around 0.2 . In the transitional range $\left(1.5 \times 10^{5}<R e<3.5 \times 10^{6}\right) S t$ may take on values as high as 0.5 . In this range the vortex shedding becomes more irregular than in the subcritical range, i.e. it is not possible to pinpoint one value for the Strouhal number. In the

\footnotetext{
*Norwegian Marine Technology Research Institute AS (MARINTEK) Trondheim, Norway.
} 
supercritical range $\left(\operatorname{Re}>3.5 \times 10^{6}\right)$ the Strouhal number is reduced to $0.1-0.3$, and the shedding again becomes regular. The degree of regularity can be seen from the power spectra of the transverse shedding force. When shedding is regular the spectrum has the shape of a narrow peak, while irregular shedding shows as a broad-banded spectrum (Sheppard and Omar, 1992)

When the cylinder is free to move in the transverse direction, yet with resistance from springs, it will respond to the (more or less) cyclic load from the vortex shedding. The magnitude of the motion depends on the Strouhal frequency and the responsiveness of the cylinder, as determined by its mass and damping coefficients and the stiffness of the springs. If the natural resonant frequency is not too far from the Strouhal frequency lock-in or synchronization may happen. In this case vortices are shed at the actual frequency of oscillation and not at the rate predicted by (1). In other words, the motion of the cylinder controls the vortex shedding. The frequency of oscillation may not be exactly equal to the expected resonant frequency of the cylinder either. This is because the process of forming and shedding vortices alters the cylinder's added mass. The added mass will be different from the still water value, causing the resonant frequency to shift somewhat too. The change in added mass can be negative or positive, causing the natural frequency to go up or down. This is demonstrated by experiments by Sarpkaya (1978) and Gopalkrishnan (1993), who found that both added mass and damping depended on frequency and amplitude. See also Vikestad (1998). For taut cables, risers and free spans of pipeline many modes of response exist (Lie and Vandiver 1998, Kleiven 2002). In the non-lock-in case the vortex-induced oscillations will be irregular, consisting of the natural frequencies of the structure plus the frequencies of the vortex-shedding taking place along the length of the structure. Waves will be seen to travel up and down the structure. Since such structures have many modes of response and many natural frequencies there are many possibilities for lock-in. When lock-in happens, the response will in general be unimodal and have the character of a standing wave. Which mode will respond depends on which natural frequency is closest to the Strouhal frequency. For structures of large vertical extent the depth variation of the current can be large. Consequently the Strouhal frequency may vary considerably over the structure's length, having the effect that several modes can be candidates for lock-in oscillation. The mechanism of frequency shifting through added-mass variation blurs the unique relationship between modeshape and still-water eigenfrequency. This means that it is not always possible to tell which mode is in action from observations of frequency. From model tests with a taut cable in sheared flow, Lie et al. (1997) report an observation of a 2nd-mode lock-in oscillation which suddenly changed into a 3rd-mode lock-in while the VIV frequency remained unchanged.

Model testing has given valuable insight in the VIV phenomenon. Still, there are unknown areas when it comes to full scale conditions, which involve high Reynolds numbers and realistic sea currents. In addition, a real riser will be disturbed by waves and the motions of the platform it is connected to. To obtain full scale data for analysis and verification of existing prediction models for VIV an instrumentation and measurement project was carried out as a part of the Norwegian Deepwater Program (NDP). Three drilling risers at fields off-shore North Norway were instrumented for measurement of riser response. The fields were Nykhøgda, Vemadomen and Helland-Hansen. Also sea current was measured at a number of depths to get the current profile for comparison with the riser response. The instrumentation was carried out by Robit Technology, now a part of CorrOcean. Accelerometers were 
chosen to be the main measuring devices, but were supplemented with sensors for rotational rate.

The paper describes the method for processing and analysis that were applied to a large number of records of riser motion and current. The purpose of the analysis was to derive quantities that would give meaningful information about the VIV phenomenon and which could be used further for calibration of VIV-prediction tools.

When accelerometers are used for measurement of VIV it must be taken into account that they will be influenced by gravity as a consequence of the angular motion of the riser, i.e. the dynamic deviations of the riser's longitudinal axis from the vertical. A central task, therefore, has been developing a method for estimating true lateral displacements from the gravity-contaminated measurements.

In principle, the same method of analysis was applied to the data from all the three offshore locations, although the instrumentation differed somewhat. The examples in this paper are based on the data from the Helland-Hansen site, as the riser there had the most extensive instrumentation.

\section{The Measurements}

The instrument system to measure riser motion at the Helland Hanssen field consisted of six instrument containers attached to the riser in positions shown in Figure 1. Each container, numbered 1 through 6 from the bottom up, included motion sensors, data acquisitioning hardware and batteries. The main sensors were accelerometers for measurement of horizontal acceleration in two orthogonal axes, $X$ and $Y$. In containers number 3, 4 and 6 the accelerometers were supplemented with sensors for measuring angular velocity about axes $X$ and $Y$.

Current was measured by a number of acoustic doppler current profilers (ADCP) mounted in a vertical mooring in the vicinity of the drilling vessel. In addition to the ADCPs a rotor-type current meter measured current near the seafloor. The raw data consisted of readings every ten minutes, representing the average values of the tenminute interval. The duration of the measuring period was about twelve weeks.

In the following we describe the task of calculating the modes of oscillation and lateral displacements of the riser from the records of acceleration and rate of rotation. Further descriptions of the Norwegian Deepwater Program and analysis of VIV are given by Olufsen and Garside (1999), Halse (2000) and Kaasen et al. (2000).

\section{Disturbance by Gravity}

An accelerometer can measure true acceleration along its sensitive axis as long as the orientation of this axis is kept constant in space. When using the accelerometer signals in calculation of the riser's lateral displacement one must take into account that the accelerometers will be influenced by gravity due to the angular motion of the riser, i.e. the dynamic deviations of the riser's longitudinal axis from the vertical. If the sensing axis of an accelerometer is tilted a small angle $\theta$ from the horizontal the vector of gravity will have a non-zero projection on the accelerometer axis. The output from the accelerometer will then be

$$
a=\ddot{x}-g \theta
$$

where $\ddot{x}$ is the true lateral acceleration and $g$ the gravitational acceleration $(\approx 9.81$ $\mathrm{m} / \mathrm{s}^{2}$ ). This shows that the accelerometer in reality is a combination of a true 


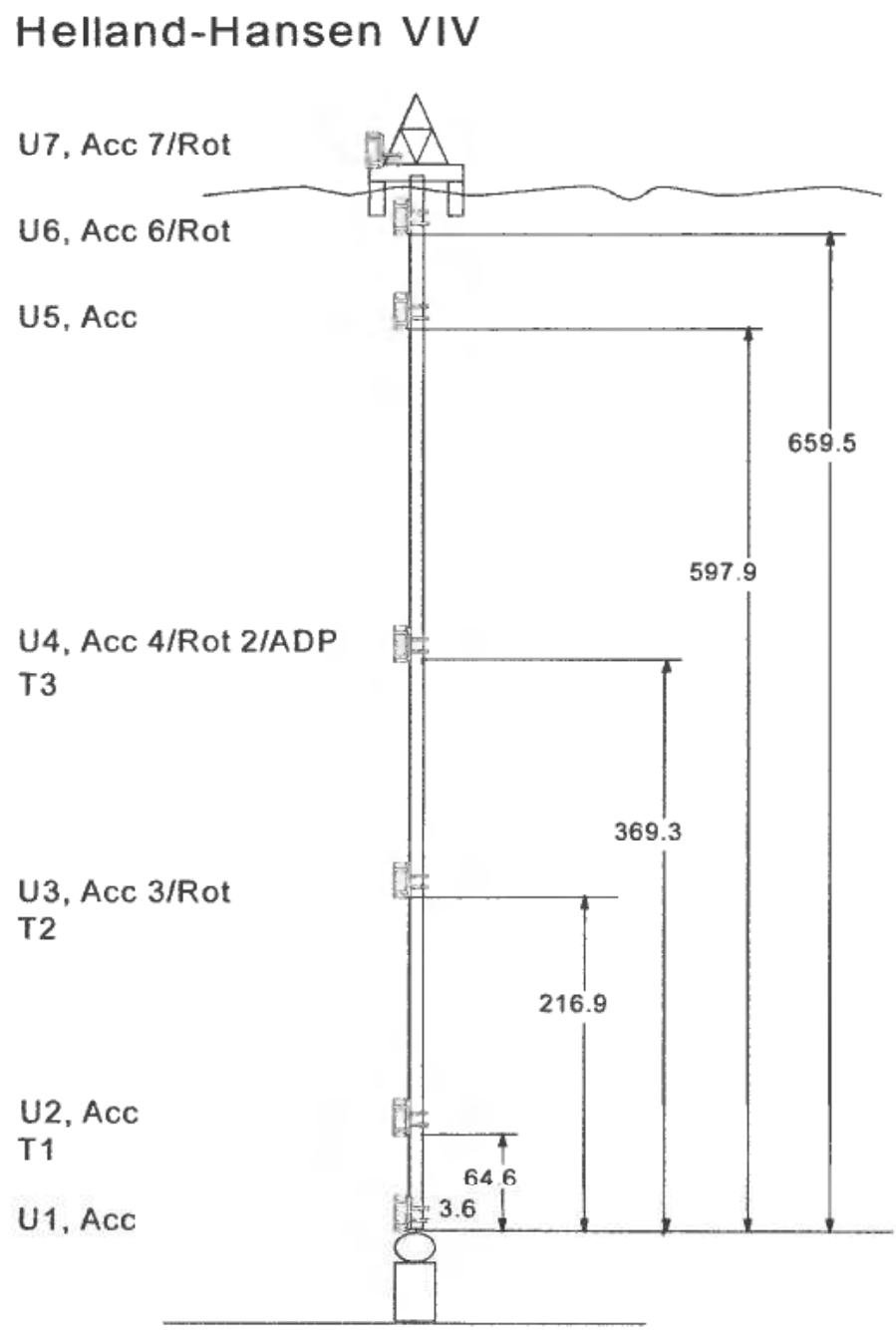

Figure 1. Locations of the six instrument containers on the Helland-Hansen drilling riser. A seventh container was installed in the drilling vessel, but was not utilized in the analysis (Figure by CorrOcean AS).

accelerometer and an inclinometer. In order to get a true acceleration signal to integrate twice into horizontal displacement one might attempt to remove the influence of gravity by some sort of signal filtering. However, this cannot be done since $\ddot{x}$ and $\theta$ contain exactly the same frequency components.

At first the accelerometer's sensitivity to inclination may seem as a fault with the device. Actually, it turns out that the inclinometer action is helpful for determining the lowest (and slowest) modes of motion from the measurements.

If the riser is moving at an eigenmode the translatory and rotary motions are linked together in a unique manner, i.e. knowing one is knowing the other. This can be extended to the case where the riser motion can be decomposed in a number of $N$ eigenmodes. In this case (subject to certain restrictions) knowing the displacements at $N$ points on the riser is equivalent to knowing the rotations at $N$ points too. The principle of modal decomposition can be exploited further as described below. 


\section{Modal Approach}

When the riser responds at one of its eigenmodes there is a unique relationship between the local tilt and the displacement at any position on the riser. This is also true when the riser response is composed of a number of different eigenmodes. This relationship can be utilized to get around the problem of disturbance by gravity.

Figure 2 shows the five first natural modes of the Helland-Hansen riser, calculated
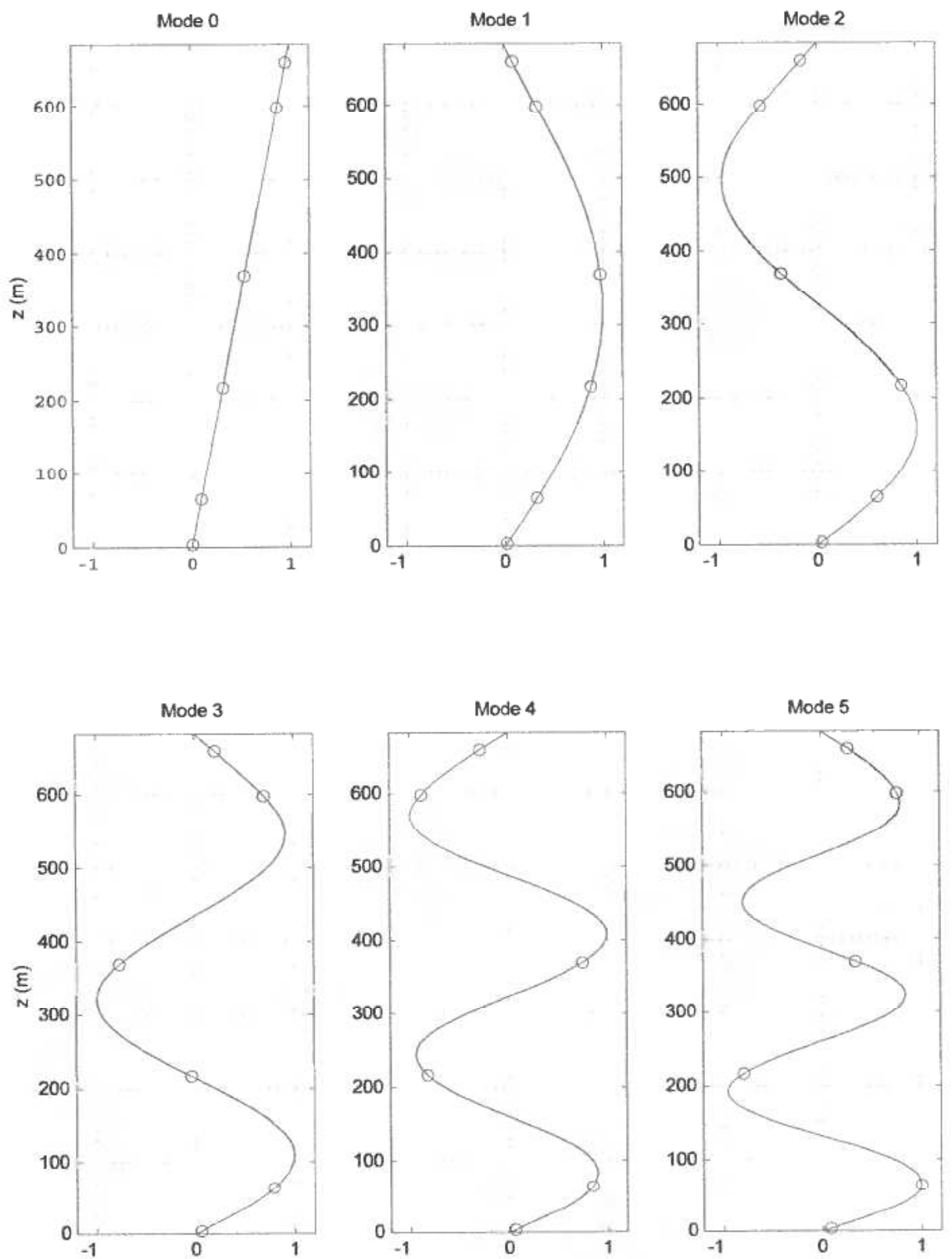

Figure 2. Modeshapes of riser displacement, calculated by FEM model. The circles show the locations of the instrument units. Units 3, 4 and 6 (from the bottom) contain rotation rate sensors in addition to the accelerometers. 
using a numerical finite elements model (RIFLEX). As is seen, the modeshapes deviate somewhat from sinusoids. This is a consequence of the increasing riser tension in the riser from the bottom up. A zeroth mode in the form of a straight line has been added to model the effect of the irregular slow motion of the dynamically positioned drilling vessel.

The modal decomposition is based on the assumption that any time-dependent shape of the riser can be expressed as a sum of eigenfunctions. For displacements in the horizontal $X$-direction, we get

$$
x(z, t)=\sum_{n=0}^{\infty} q_{n}(t) d_{n}(z)
$$

where $z$ denotes the vertical position on the riser (chosen positive upwards, zero at lower end) and $d_{n}(z)$ is the $n$-th eigenfunction. The variable $q_{n}(t)$ is the instantaneous magnitude (weight factor, modal participation factor, generalized coordinate) of the $n$-th mode. For displacements along the direction of $Y$ there is an identical expression.

The inclination of the riser, i.e. the angular deviation from the vertical line, is obtained by differentiating (3) with respect to $z$. This gives

$$
\theta(z, t)=\sum_{n=0}^{\infty} q_{n}(t) r_{n}(z)
$$

where

$$
r_{n}(z)=\frac{d}{d z} d_{n}(z)
$$

is the eigenfunction of rotation which corresponds to $d_{n}(z)$.

For displacements along $X$ the method for estimating true riser displacements is described as follows:

Let $z_{1}, z_{2}, z_{3}, z_{4}, z_{5}, z_{6}$ denote the positions of the instrument units on the riser. Inserting these coordinates in equations (3) and (4) gives the displacement and angle at the instrumented positions. We want to express this in matrix notation and define the matrices

$$
\begin{aligned}
\mathbf{x} & =\left[x\left(z_{1}\right), x\left(z_{2}\right), x\left(z_{3}\right), x\left(z_{4}\right), x\left(z_{5}\right), x\left(z_{6}\right)\right]^{T} \\
& =\left[x_{1}, x_{2}, x_{3}, x_{4}, x_{5}, x_{6}\right]^{T} \\
\mathbf{q} & =\left[q_{0}, q_{1}, q_{2}, \ldots, q_{N}\right]^{T} \\
\overline{\mathbf{d}}_{n} & =\left[d_{n}\left(z_{1}\right), d_{n}\left(z_{2}\right), d_{n}\left(z_{3}\right), d_{n}\left(z_{4}\right), d_{n}\left(z_{5}\right), d_{n}\left(z_{6}\right)\right]^{T}, n=0,1, \ldots, N \\
\mathbf{D} & =\left[\overline{\mathbf{d}}_{0}, \overline{\mathbf{d}}_{1}, \overline{\mathbf{d}}_{2}, \ldots, \overline{\mathbf{d}}_{N}\right]
\end{aligned}
$$

Here it is assumed that modes above the $N$-th mode do not contribute to the response. The mapping from modal weights to displacements at the given coordinates now becomes

$$
\mathbf{x}=\mathbf{D q}
$$

Likewise, the mapping from modes to angles can be written

$$
\boldsymbol{\theta}=\mathbf{R q}
$$

where $\boldsymbol{\theta}$ and $\mathbf{R}$ are defined in exact analogy with $\mathbf{x}$ and $\mathbf{D}$. 
Defining the vector of accelerometer signals,

$$
\mathbf{a}=\left[a_{1}, a_{2}, a_{3}, a_{4}, a_{5}, a_{6}\right]^{T}
$$

the model for these measurements becomes (cf. equation (2)):

$$
\mathbf{a}=\ddot{\mathbf{x}}-\mathbf{g} \boldsymbol{\theta}+\boldsymbol{\xi}_{a}=\mathbf{D} \ddot{\mathbf{q}}-\mathbf{g} \mathbf{R q}+\xi_{a}
$$

Here, $\xi_{a}$ is a vector of measurement noise, which is necessary to make the picture complete. Note that 'noise' does not have to be interpreted as physical noise or disturbance. This term may equally well include any cause of measurement error, such as general sensor imperfection or inaccuracy.

Further, considering the measurements of rate of rotation: Let $\rho_{1}, \rho_{2}$ and $\rho_{3}$ be the signals from the rotational rate sensors in instrument units 3, 4 and 6 . We get the relation

$$
\rho=\left[\begin{array}{c}
\rho_{1} \\
\rho_{2} \\
\rho_{3}
\end{array}\right]=\left[\begin{array}{c}
\dot{\theta}_{3}+\xi_{\rho 1} \\
\dot{\theta}_{4}+\xi_{\rho 2} \\
\dot{\theta}_{6}+\xi_{\rho 3}
\end{array}\right]=\mathbf{S} \dot{\theta}+\xi_{\rho}=\mathbf{S R \dot { q }}+\xi_{\rho}
$$

where $\xi_{\rho}=\left[\xi_{\rho 1}, \xi_{\rho 2}, \xi_{\rho 3}\right]^{T}$ is measurement noise. $\mathbf{S}$ acts as a sifting matrix that sifts out the rotation rates that are measured, i.e.

$$
\mathbf{S}=\left[\begin{array}{l}
001000 \\
000000 \\
000001
\end{array}\right]
$$

It is convenient to do the calculations in the frequency domain. Firstly, frequencydomain techniques are efficient from a computational view. Secondly, filtering and removal of unwanted signal components are easy in the frequency domain. Applying Fourier transformation to equations (10) and (11) gives

$$
\begin{aligned}
& \mathbf{a}=\left(-\omega^{2} \mathbf{D}-g \mathbf{R}\right) \mathbf{q}+\xi_{a} \\
& \mathbf{\rho}=-j \omega \mathbf{S R q}+\xi_{\rho}, \quad j=\sqrt{-1}
\end{aligned}
$$

where it is to be understood that the variables now are functions of frequency.

Defining the combined vectors $m=\left[\mathbf{a}^{T}, \rho^{T}\right]^{T}$ and $\xi=\left[\xi_{a}^{T}, \xi_{\rho}^{T}\right]^{T}$, equation (13) can be written as

$$
\mathbf{m}(\omega)=\mathbf{G}(\omega) \mathbf{q}(\omega)+\xi
$$

where the matrix $\mathbf{G}(\omega)$, defined as

$$
\mathbf{G}(\omega)=\left[\begin{array}{c}
-\omega^{2} \mathbf{D}-\mathbf{g} \mathbf{R} \\
-j \omega \mathbf{S R}
\end{array}\right],
$$

is the matrix transfer function from the vector of modal weights $q$ to the measurement vector $\mathbf{m}$.

We want to determine the modal vector $\mathbf{q}$ from the measurement $\mathbf{m}$. To be able to do this, $\mathbf{q}$ must contain at most nine components, since there are nine measurements in $\mathbf{m}$ (six accelerations and three angular velocities). However, trying to estimate as many as nine modal variables may lead to noise-sensitive results. This is because the 
three measurements of angular velocity cannot be regarded as fully independent information since they are taken at the positions on the riser where there are accelerometers already. The angular rate meters must rather be regarded as backup sensors for the accelerometers.

When fewer than nine modes are estimated equation (14) can be solved in the least squares sense, i.e. by minimising the quadratic scalar expression

$$
J(\mathbf{q})=[\mathbf{m}(\omega)-\mathbf{G}(\omega) \mathbf{q}(\omega)] * \mathbf{W}[\mathbf{m}(\boldsymbol{\omega})-\mathbf{G}(\boldsymbol{\omega}) \mathbf{q}(\omega)]
$$

with respect to $\mathbf{q}$. Here, $\mathbf{W}$ is a diagonal weighting matrix, which is necessary when comparing measurements of different type and magnitude. The asterisk denotes the combined operation of matrix transposition complex conjugation. Theoretically, the elements of $\mathbf{W}$ should be chosen as the standard deviations of the elements of the measurement errors. As this information was difficult to assess, the following weight matrix was used instead:

$$
\mathrm{W}=\operatorname{diag}\left(\sigma_{a}^{-2}, \sigma_{a}^{-2}, \sigma_{a}^{-2}, \sigma_{a}^{-2}, \sigma_{a}^{-2}, \sigma_{a}^{-2}, \sigma_{\rho}^{-2}, \sigma_{\rho}^{-2}, \sigma_{\rho}^{-2}\right)
$$

Here $\sigma_{a}$ is the largest standard deviation found amongst the members of $a$, and $\sigma_{\rho}$ is the largest standard deviation of angular velocity $\rho$. Minimising equation (16) with respect to $q$ gives the least squares solution for the the modal weights (Goodwin and Payne, 1977):

$$
\hat{\mathbf{q}}(\omega)=\left[\mathbf{G}(\omega)^{*} \mathbf{W G}(\omega)\right]^{-1} \mathbf{G}(\omega)^{*} \mathbf{W m}(\omega)
$$

Finally, using equation (7) we get the estimate for the horizontal displacements, i.e.

$$
\hat{\mathbf{x}}(\omega)=\operatorname{Dâ}(\omega)
$$

Inverse Fourier transformation takes the displacements to the time domain.

The estimation for the $Y$ dimension is analogous. The only difference in the equations is that the sign of the matrix $\mathbf{R}$ must be changed.

A comment on the pre-calculated modeshapes (Fig. 2): These modeshapes are the eigenvectors of an undamped numerical model. This results in real modeshapes. In general, with non-zero damping, the modeshapes will be complex, cf. Hoen and Moe (1999). For complex modes, the phase of oscillation will vary along the riser. For a real mode the phase is constant. The main reason why the damping cannot be predicted is the fact that the damping will vary along the riser and depend on the current speed, the frequency of vibration and the local amplitude. See Sarpkaya (1978) and Gopalkrishnan (1993). In the case of lock-in the damping must be negative in some parts of the riser. Since no reliable estimate of the damping could be obtained a priori it could just as well be set to zero. In addition to the uncertainty about the damping, the unknown variation of added mass along the riser will also contribute with some error.

Although the calculated real mode-shapes in Figure 2 cannot be expected to be exactly equal to the true shapes, this does not mean that they cannot be used in the calculation. This is clearly demonstrated by the results in Figures 4 6. Actually, the method of estimation can be used with any set of functions that have the ability to span the space of riser vibration. Of course it is convenient to have functions that approximate the true shapes. Otherwise a large number of functions could be needed. (It should be mentioned that since lock-in motion is not a linear dynamic process, eigenmodes, in the proper meaning of the word, do not exist). 
Current Data: 1998.05.05 02:00

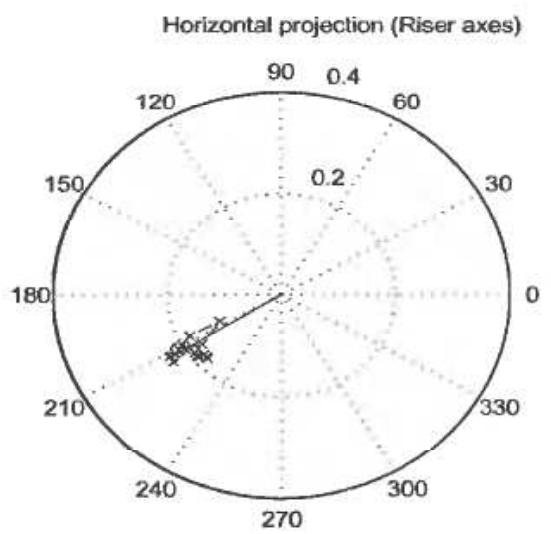

Principal Dir $=-148 \mathrm{deg}$

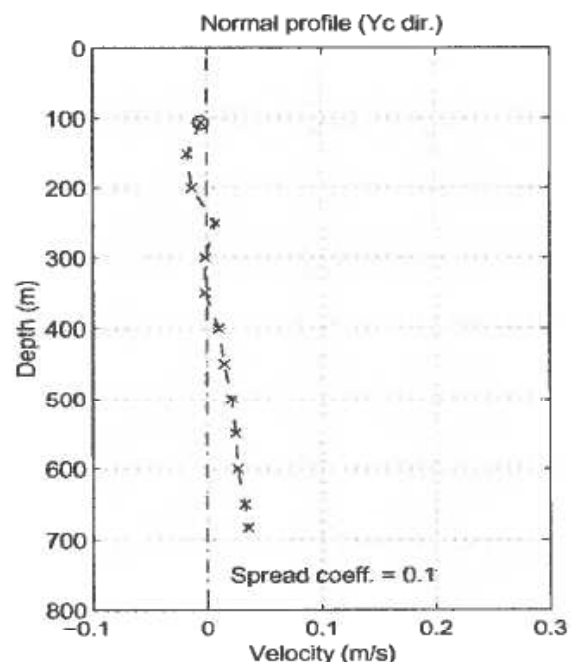

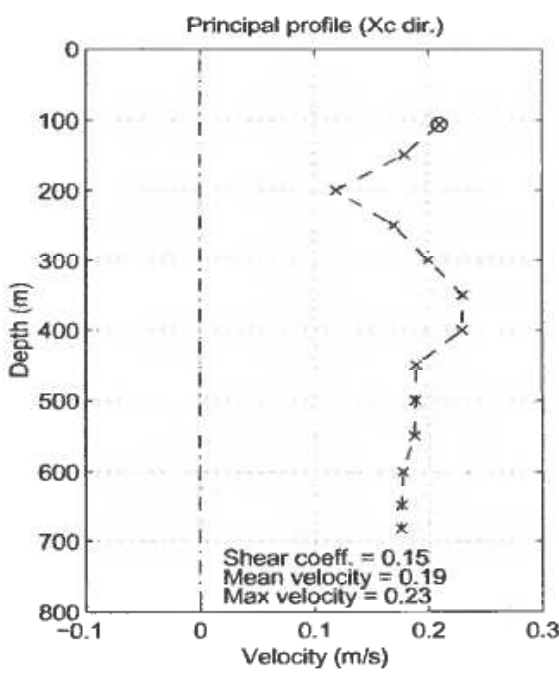

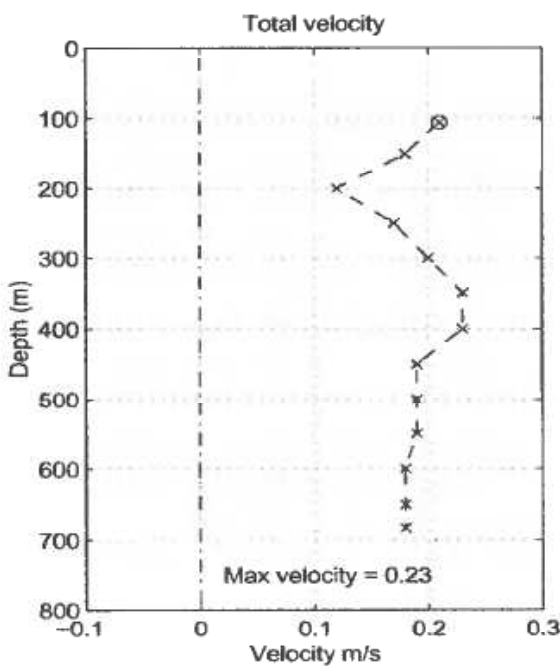

Figures 3. (Sheet 1) Current profile and data.

\section{Mode Selection and Filtering}

To use the least squares estimation technique described above, the modes one expects to find must be pre-selected. CorrOcean's report from the data collection project showed that modes up to the fifth occurred - the fifth only on rare occasions. While it was considered possible to estimate six modes simultaneously (the number of instrumented locations being six), this number was reduced to five to gain some extra robustness. Most of the time the andysis was done with modes $\{0,1,2,3,4\}$. For occasions when the fifth mode was present in the response and had to be included in the analysis, one of the others was omitted, most often the 0th mode.

To prevent the estimates of modal weights to be disturbed by signal components outside the frequency range that was relevant for the selected modes, the measurements were bandpass-filtered before use (Filtering the results would be equivalent). 

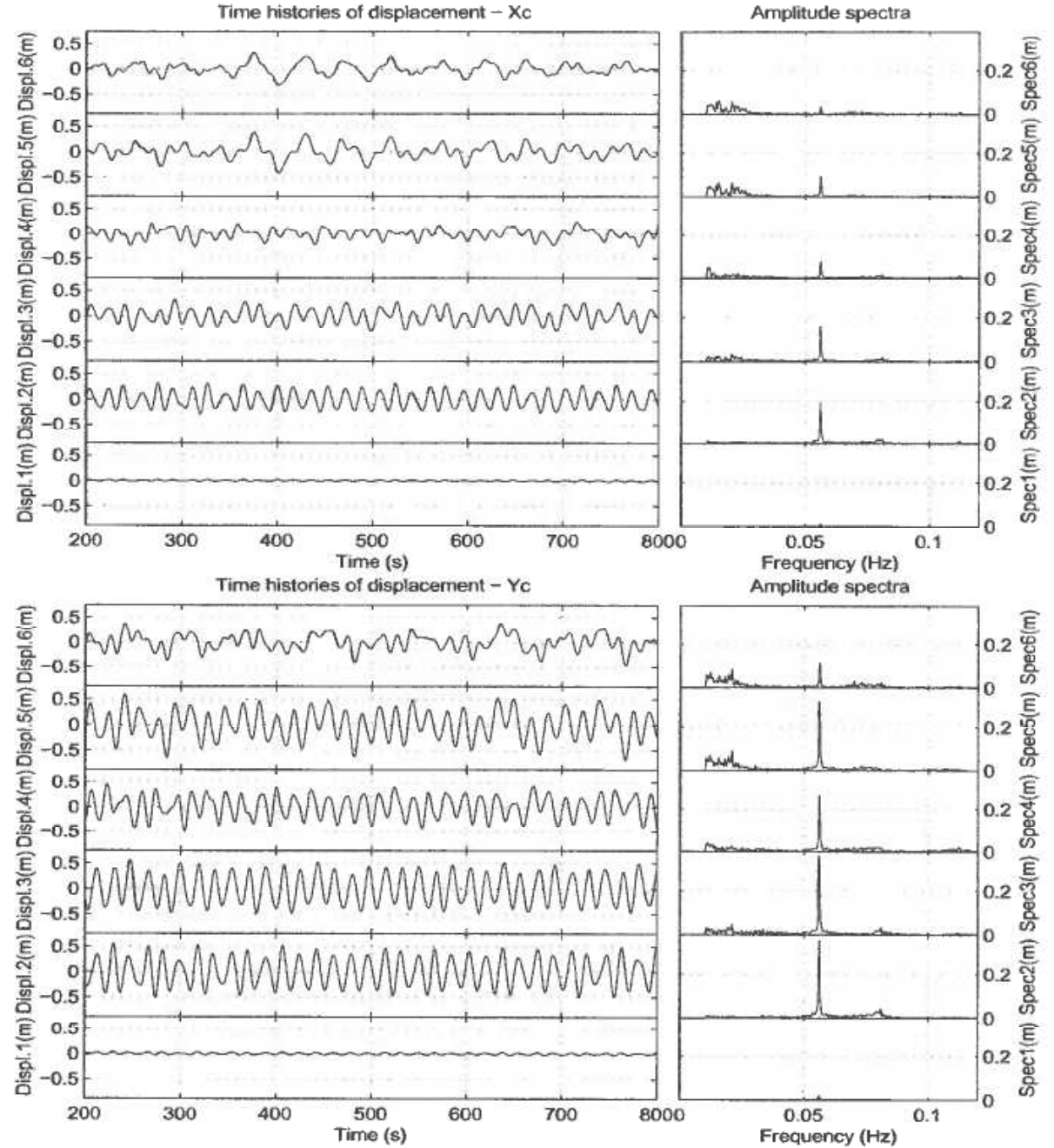

Figure 4. (Sheet 2) Estimated displacement and amplitude spectrum at instrument locations.

The cut-off frequencies of the bandpass filter varied with the chosen set of modes, but typically, the pass-band range was set to $0.01-0.16 \mathrm{~Hz}$. The upper cut-off frequency could have been set to a lower value, preventing the wave-induced motion components to slip through. However, this was never a problem, as the least-squares estimation method performed well in every instance and had never any difficulty in producing results that seemed trustworthy.

\section{Analysis of Current}

The purpose of the current analysis was to find the best 2-dimensional representation of the measured profile as a preparation for the model verification. In addition a number of characterising parameters were calculated.

In a 2-D (uni-directional) current profile the VIV response will mainly occur in a plane that is perpendicular to the direction of the current (i.e. cross-flow response). Most VIV-prediction programs assume 2-D current. Real current profiles are 3-D, 

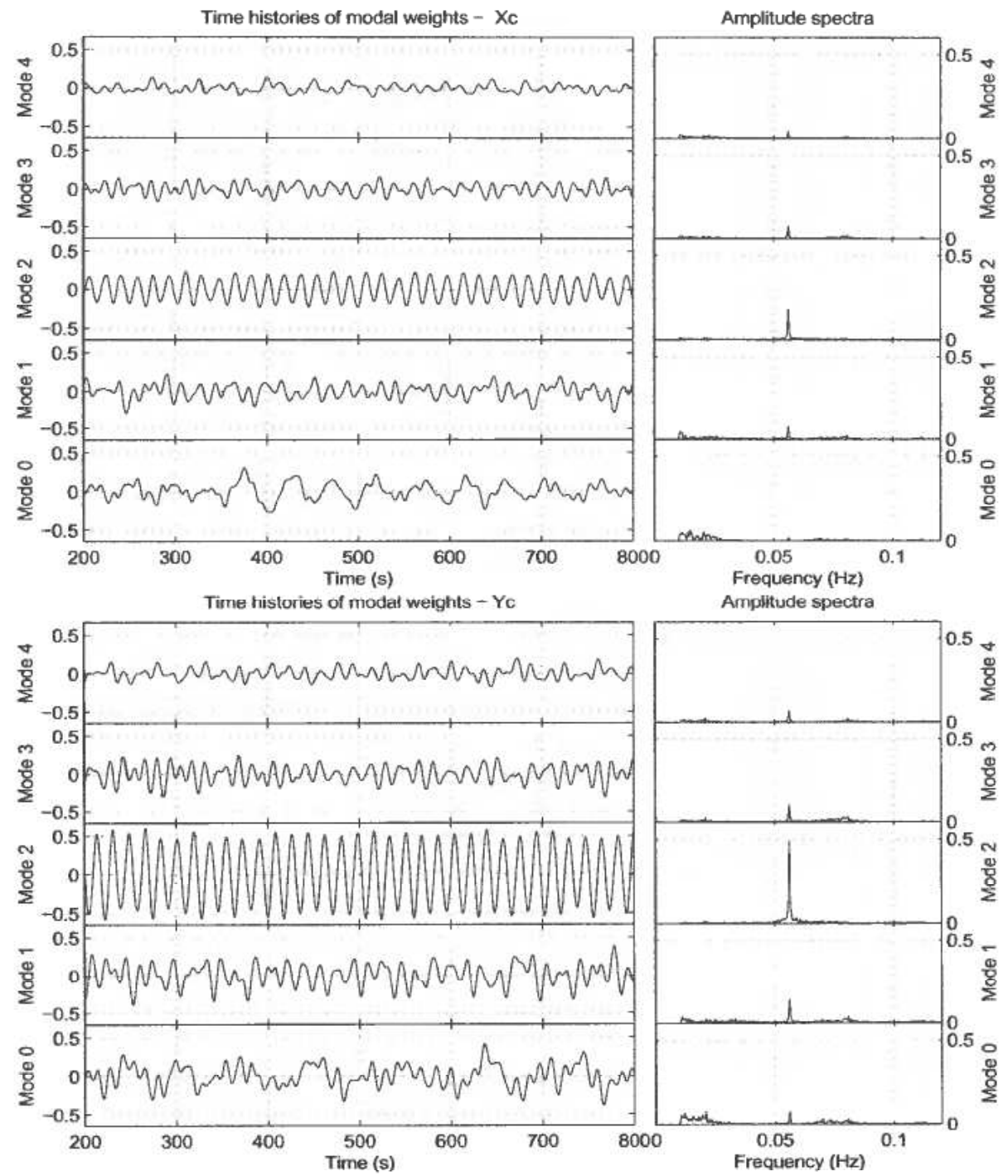

Figure 5. (Sheet 3) Time series and amplitude spectra of modal weights.

but often with a dominating, or main, direction. The riser may therefore behave as if the profile be 2-D, at least in some cases.

To make comparison of results easy between different cases of different main current directions, the riser measurements were transformed to a new coordinate system in which the $X$-axis coincided with the main direction of the current. In the new axes, the $X$ response of the riser will be the in-line response, while the $Y$ response will be the cross-flow response.

The main current direction was defined as the orientation of the $X Z$ plane onto which the total projection of all the measured current velocity vectors be maximum.

Some characteristic parameters were calculated from the transformed current profile. The spreading coefficient informed of how much the current profile deviated from 2-D. The shear coefficient quantified the degree of vertical non-uniformity. For definition of these parameters, see Kaasen et al. (2000). 

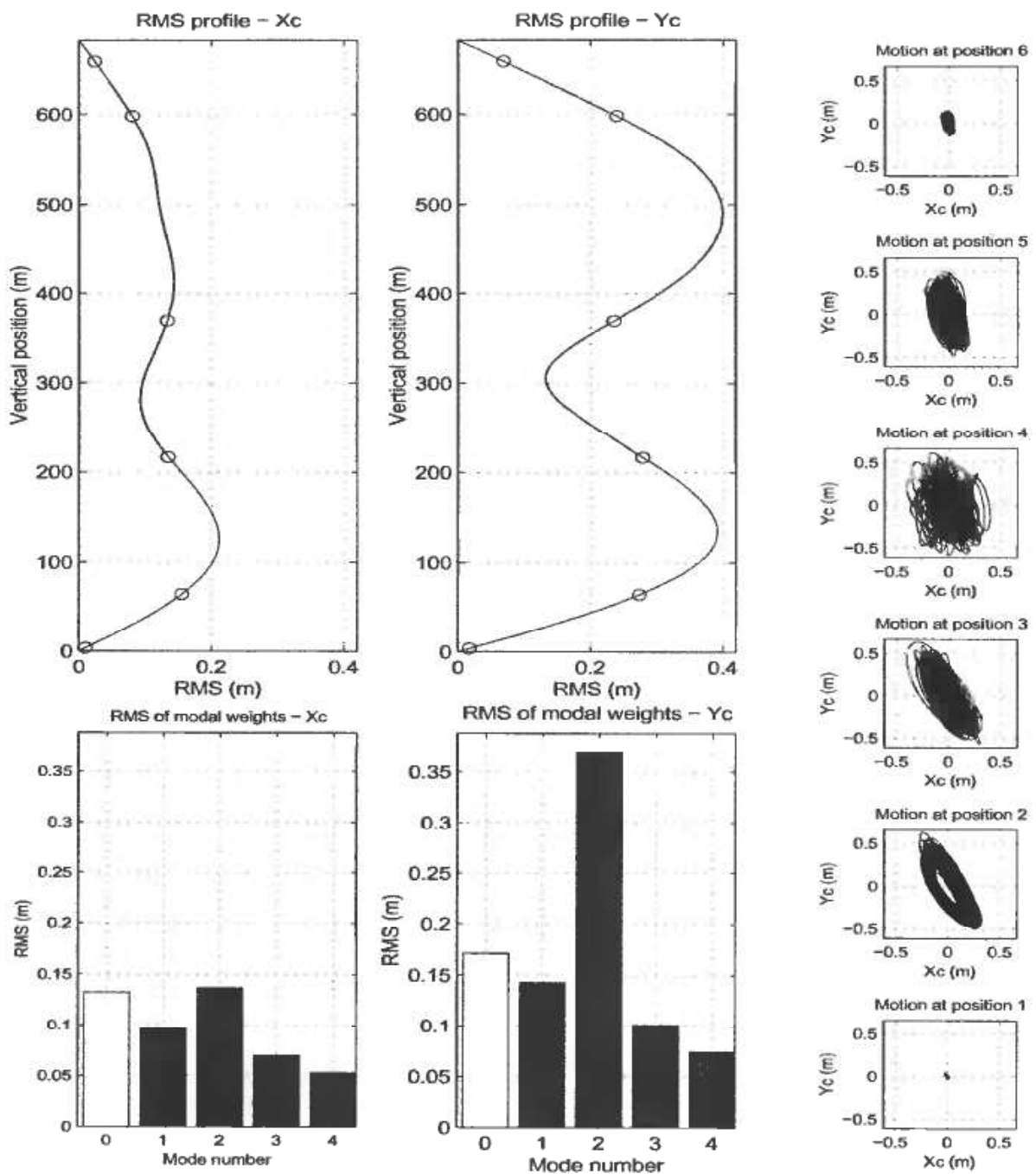

Figure 6. (Sheet 4) RMS-profile of riser displacement, modal weights and xy-plots of riser displacements at instrument locations.

\section{Calculation and Presentation Using MATLAB}

For standard tasks in the field of marine engineering and research MARINTEK uses a variety of computer programs, many of which are developed in-house. Typical examples are programs for simulation and analysis of the behaviour of ships, offshore platforms, offshore cranes, mooring cables and risers subjected to disturbances from the metocean environment. The majority of these programs are written in Fortran. For isolated, special tasks like the present problem of estimating riser VIV displacements from a given set of measured variables, no ready-made software was applicable.

As often before in similar situations MATLAB was chosen for the task. In the present case, MATLAB was particularly well suited, since the problem was naturally formulated in terms of matrices, being the basic variables in MATLAB. Moreover, a variety of matrix functions are available.

For example, the problem of finding the main current direction (see above) is 
easily solved using the eigs function in MATLAB. Assume current is measured at $N$ depths. Defining the current velocity at depth $i$ as a vector $\mathbf{u}_{i}=\left[u_{i}, v_{i}\right]^{\prime}$ (apostrophe denotes transpose) and the current profile array $\mathbf{U}=\left[\mathbf{u}_{1}, \mathbf{u}_{2}, \ldots, \mathbf{u}_{N}\right]$, the unit vector in the main current direction is simply found as the first output argument in $[\mathbf{E}, \mathbf{D}]=\operatorname{eigs}\left(\mathbf{U}^{*} \mathbf{U}^{\prime}, 1\right)$.

Beside the matrix-handling capability, the Fast Fourier Transform function fft was a cornerstone in the calculation, together with the inverse transformation function ifft. The feasibility of using the frequency domain as an arena for practical time series analysis was made possible by Cooley and Tukey (1965) who invented the FFT algorithm. While it is possible to solve the problem of calculating VIV displacement using discrete-time methods, the frequency domain method is much simpler. Besides, it is considerably easier to do noise filtering in the frequency domain.

The way the results from the calculation were presented was an important issue in the project. Printed reports containing graphs and tables were to be distributed to the members of the NDP group. In order to keep the number of pages at a manageable number it was important that a compact way of presenting the results could be found. For the graphical presentation customisation was necessary. MATLAB's object-oriented graphics system offered what was needed. For each recorded set of riser and current measurements the result of the analysis was presented on four sheets of paper, shown as Figures 3 to 6. On the first sheet (Fig. 3) the current was presented. The second sheet (Fig. 4) shows the calculated VIV displacement at the six instrumented locations on the riser. The displacements are shown as time traces and amplitude spectra. The third sheet is similar to the second, but shows the modal weights. The fourth sheet shows the riser's rms-profile, the rms-values of the modes as bar plots and XY-trajectories of the motion at the instrumented locations.

In addition to the graphical presentation tables of statistical information were made.

\section{An Analysis Example}

The Helland-Hansen data consists of than 916 recorded sets of riser motion and current. Each record of riser measurement has a length of 34 minutes and consists of 4096 samples. Based on a screening of the raw data, 357 sets were selected for analysis. In what follows, the method for analysing and identifying VIV is exampled by one case. This case is a typical lock-in case, with an almost pure 2nd mode VIV.

Fig. 3 presents the current situation. The figure is split into four plots. The upper left plot shows a polar plot of the current flow in a horizontal projection. The principal direction of the current is shown as a radial line. The upper right plot presents the current profile projected onto the principal plane, i.e. vertical plane extending in the main direction (along axis $X$ ). The plot on the lower left is the normal projection of the current profile (perpendicular to $X$ ), i.e. the spreading profile. The lower right plot shows the total speed profile.

Using the estimator described above, modal weights and displacements were estimated. For the six instrumented locations on the riser, Figure 4 shows the estimated displacements in the cross-flow direction in the form of time traces and amplitude spectra. As can be seen the 2nd mode dominates completely, but there are some irregular low frequency action to be seen and some blurred energy around the third natural frequency. The low frequency motion is most prominent toward the top of the riser indicating that its source is the slowly varying motion of the dynamically positioned drilling vessel. 
The estimated modal weights (modes 1-4 plus mode 0) are shown in Figure 5. The result demonstrates that the estimator works well (at least for the case shown). Mode 2 clearly dominates. The bulk of vessel-induced low frequency motion is picked up by the zeroth ('garbage') mode, as intended. There are some small amounts of mode 2's frequency to be seen on the other mode estimates. This is natural, since for an arbitrary distribution of current-dependent excitation along the riser, the response cannot be expected to be purely unimodal.

Using the estimated modal weights (participation factors) and pre-calculated modeshapes the rms profile of the riser is calculated and shown in Figure 6. In this calculation the zeroth mode was excluded, since it is not a VIV motion. The figure shows clearly the dominance of the second mode.

\section{Conclusion}

Data from full-scale measurements are necessary for verification of theoretical models and tools for prediction of VIV. For such verification the riser's lateral motions must be estimated and compared with measured depth-varying current.

A method for estimating the VIV displacement of a marine riser from measurements of acceleration and rate of rotation is developed. A fundamental problem with using accelerometers to measure riser motion is that the signals may be significantly influenced by gravity due to the angular motion of the riser. On the other hand, it is this gravitational 'disturbance' that makes detection of low frequency motion possible. The estimation method can handle the influence of gravity utilizing a modal approach in combination with a least-squares technique. Also the method can deal with cases where different instrumentation is used in different locations on the riser (i.e. not every instrument container included a rotational-rate device). The estimator operates in the frequency domain, which is efficient from a computational view. The estimator uses pre-calculated modeshapes, which must be derived from a theoretical model of the riser. An example shows that the estimator works well.

Except computing the modeshapes for the riser, all calculation and presentation were done with MATLAB, which proved to be an excellent tool for the task.

\section{Acknowledgement}

The work described in this paper was performed as a part of the Norwegian Deepwater Programme (NDP). Partners in the NDP group have been BP Amoco, Conoco, Exxon, Mobil, Norsk Hydro, Saga, Shell, and Statoil. The authors thank CorrOcean for permission to use Figure 1.

\section{Reference}

BLevins, R. D. (1990). Flow-Induced Vibration, 2nd Ed., Van Nostrand Reinhold, New York.

CoOley, J. W. and Tukey, J. W. (1965) An Algorithm for the Machine Calculation of Complex Fourier Series. Math of Computation, Vol. 19, pp. 297-301.

Goodwin, G. C. and PAYNE, R. L. (1977). Dynamic System Identification. Experiment Design and Data Analysis. Academic Press, New York.

GopalkrishnaN, R. (1993). Vortex-Induced Forces on Oscillating Bluff Cylinders. Doctoral Thesis, Dept. of Ocean Engineering, Massachusetts Institute of Technology and Woods Hole Oceanographics Institution, USA.

HALSE, K. H. (2000). Norwegian Deepwater Program. Improved VIV Prediction, Paper 11996, Offshore Technology Conference. Houston, USA. 
HoEN, C. and MOE, G. (1999), Modal decomposition of measured vortex induced response of drilling rises, Proceedings of the Ninth International Offshore and Polar Engineering Conference (ISOPE), Brest, France.

KaAsen, K. H., Lie, H., Solaas, F. and Vandiver, J. K. (2000). Norwegian Deepwater Program. Analysis of Vortex-Induced Vibrations of Marine Risers Based on Full-Scale Measurements. Paper 11997, Offshore Technology Conference, Houston, USA.

KLEIVEN, G. (2002). Identifying VIV vibration modes by use of the Empirical Orthogonal Functions technique. Proceedings of the 21th International Conference on Offshore Mechanics and Arctic Engineering, Oslo, Norway.

Lie, H., Larsen, C. M. and Vandiver, J. K. (1997). Vortex Induced Vibrations of Long Marine Risers; Model Test in a Rotating Rig. 16th Int. Conf. Offshore Mechanics and Arctic Engineering, Yokohama, Japan.

LIE, H. and VANDIVER, J. K. (1998): VIV Model Test of a Bare and Staggered Buoyancy Riser in a Rotating Rig, Paper OTC 8700, Offshore Technology Conference, Houston.

Olufsen, A and Garside, R (1999). Riser \& Mooring Research Activities Within the Norwegian Deepwater Program, Deep Offshore Technology Conference, Stavanger, Norway.

SarpKaya, T (1978). Fluid Forces on Oscillating Cylinders, J. of The Waterway. Port. Coastal and Ocean Division, 104 (WW4), August 1978.

SHEPPARD, D. M. and OMAR, A. F. (1992). Vortex-induced loading on offshore structures: A selective review of experimental work. Paper No. 6817, Proc. 24th Offshore Technology Conference, Houston, USA.

VANDIVER, J. K. (1993). Dimensionless parameters important to the prediction of vortexinduced vibration of long, flexible cylinders in ocean currents, J. of Fluids and Structures 7.

VANDIVER, K. (1998): Research Challenges in the Vortex-Induced Prediction of Marine Risers, Paper OTC 8698, Offshore Technology Conference, Houston.

VikestaD, K. (1998): Multi-Frequency Response of a Cylinder Subjected to Vortex-Shedding and Support Motions, Doctoral Thesis, Norwegian University of Science and Technology, Trondheim. 